Cite this: Phys. Chem. Chem. Phys., 2011, 13, 5994-5999

\title{
Mechanically activated rupture of single covalent bonds: evidence of force induced bond hydrolysis $\dagger$
}

\author{
Sebastian W. Schmidt, ${ }^{a b c}$ Alfred Kersch, ${ }^{a}$ Martin K. Beyer ${ }^{* b}$ and \\ Hauke Clausen-Schaumann*ac
}

Received 23rd November 2010, Accepted 27th January 2011

DOI: $10.1039 / \mathbf{c 0 c p 0 2 8 2 7 d}$

We have used temperature-dependent single molecule force spectroscopy to stretch covalently anchored carboxymethylated amylose (CMA) polymers attached to an amino-functionalized AFM cantilever. Using an Arrhenius kinetics model based on a Morse potential as a one-dimensional representation of covalent bonds, we have extracted kinetic and structural parameters of the bond rupture process. With $35.5 \mathrm{~kJ} \mathrm{~mol}^{-1}$, we found a significantly smaller dissociation energy and with $9.0 \times 10^{2} \mathrm{~s}^{-1}$ to $3.6 \times 10^{3} \mathrm{~s}^{-1}$ also smaller Arrhenius pre-factors than expected for homolytic bond scission. One possible explanation for the severely reduced dissociation energy and Arrhenius pre-factors is the mechanically activated hydrolysis of covalent bonds. Both the carboxylic acid amide and the siloxane bond in the amino-silane surface linker are in principle prone to bond hydrolysis. Scattering, slope and curvature of the scattered data plots indicate that in fact two competing rupture mechanisms are observed.

\section{Introduction}

Chemical bonds control the stress and wear resistance and thus the toughness and durability of synthetic and natural materials. ${ }^{1}$ To understand material failure it is therefore essential to understand the breakdown of individual chemical bonds. Like most chemical reactions, the scission of chemical bonds requires activation energy. This energy can be provided in the form of light, thermal, electrical or mechanical energy. When it comes to material failure, mechanical activation plays a crucial role, as materials often bear considerable mechanical forces in everyday life. Moreover, mechanical activation can be used in natural products chemistry, the chemistry of solids, and even in organic synthesis, where force can be used as a control parameter to steer a reaction to a desired end point. ${ }^{2-8}$ With the advancement of single molecule techniques, rupture forces of individual molecular bonds have become experimentally accessible, ${ }^{9-13}$ and recently there has been an increasing number of studies focusing on the mechanical stability of covalent chemical

${ }^{a}$ Munich University of Applied Sciences, Department of Precision- and Micro-Engineering, Engineering Physics, Lothstr. 34, 80335 Munich, Germany.E-mail: clausen-schaumann@hm.edu;

Fax: + 49 (0)89-1265-1480; Tel: + 49 (0)89-1265-1671

${ }^{b}$ Institut für Physikalische Chemie, Christian-Albrechts-Universität zu Kiel, Olshausenstr. 40, 24098 Kiel, Germany.

E-mail: beyer@phc.uni-kiel.de; Fax: +49 (0)431-880-2830;

Tel: + 49 (0) 431-880-2831

${ }^{c}$ Center for Nanoscience (CeNS), Geschwister-Scholl-Platz 1, 80539 Munich, Germany

$\dagger$ Electronic supplementary information (ESI) available: Details of the data analysis including theoretical background and description of the fitting procedure. See DOI: $10.1039 / \mathrm{c} 0 \mathrm{cp} 02827 \mathrm{~d}$ bonds. ${ }^{4,14-21}$ In order to understand the underlying chemical reactions at the molecular level, it is necessary to determine structural and kinetic parameters, like depth and width of the binding potential, as well as the Arrhenius pre-factor and compare these parameters to thermodynamic data as well as quantum chemical modelling. ${ }^{22-28}$ So far, however, it has been difficult to extract these parameters from single molecule force measurements of covalent bonds.

In a recent study, ${ }^{18}$ we have stretched single carboxymethylated amylose (CMA) polymers attached to an amino-functionalized AFM cantilever. The systematic variation of force-loading rates over several orders of magnitude revealed a thermally activated rupture process following Arrhenius type kinetics: $k^{\text {off }}=A \exp \left[-E_{\mathrm{a}}(f) /\left(k_{\mathrm{B}} T\right)\right]$, with a force-dependent activation energy $E_{\mathrm{a}}(f)$, where the bond lifetime $\tau=1 / k^{\text {off }}$ decreased with increasing force and the observed bond rupture force increased almost logarithmically with the force-loading rate $\mathrm{d} f / \mathrm{d} t$. Nevertheless, even a variation of force-loading rates over three orders of magnitude did not provide structural and kinetic information about the binding potential, since the three parameters which describe the dissociation kinetics could not be unambiguously extracted from the experimental data. In the present study, we have therefore chosen an extended approach: together with the force-loading rate, we have varied the temperature which is an additional control parameter directly entering the exponent in the Arrhenius equation. Furthermore, rather than using a least square fit algorithm, which assumes Gaussian probability distributions, we have employed the maximum likelihood method, which can be used to fit also non-Gaussian probability distributions, ${ }^{29}$ like the 
rupture force distribution of the Arrhenius kinetics model used here. With temperature as an additional parameter, and with a fit algorithm which takes into account the correct (non-Gaussian) shape of the loading rate-dependent bond rupture probabilities, we have now been able to obtain the parameters determining the unbinding kinetics of the system. A comparison of these results to thermodynamic and spectroscopic data as well as theoretical modelling indicates that a possible rupture mechanism in this case could be the mechanically activated hydrolysis of the amide or the siloxane bond in the surface linker.

\section{Results and discussion}

A schematic representation of the covalent anchoring, with the CMA polymer attached to the substrate and the AFM cantilever via amino-linkers, is shown in Scheme 1.

A typical force-extension curve of a single CMA polymer at room temperature in PBS $(150 \mathrm{mM} \mathrm{NaCl}, \mathrm{pH} 7.4)$ is displayed in Fig. 1. As described elsewhere, ${ }^{14,18,30-33}$ the characteristic

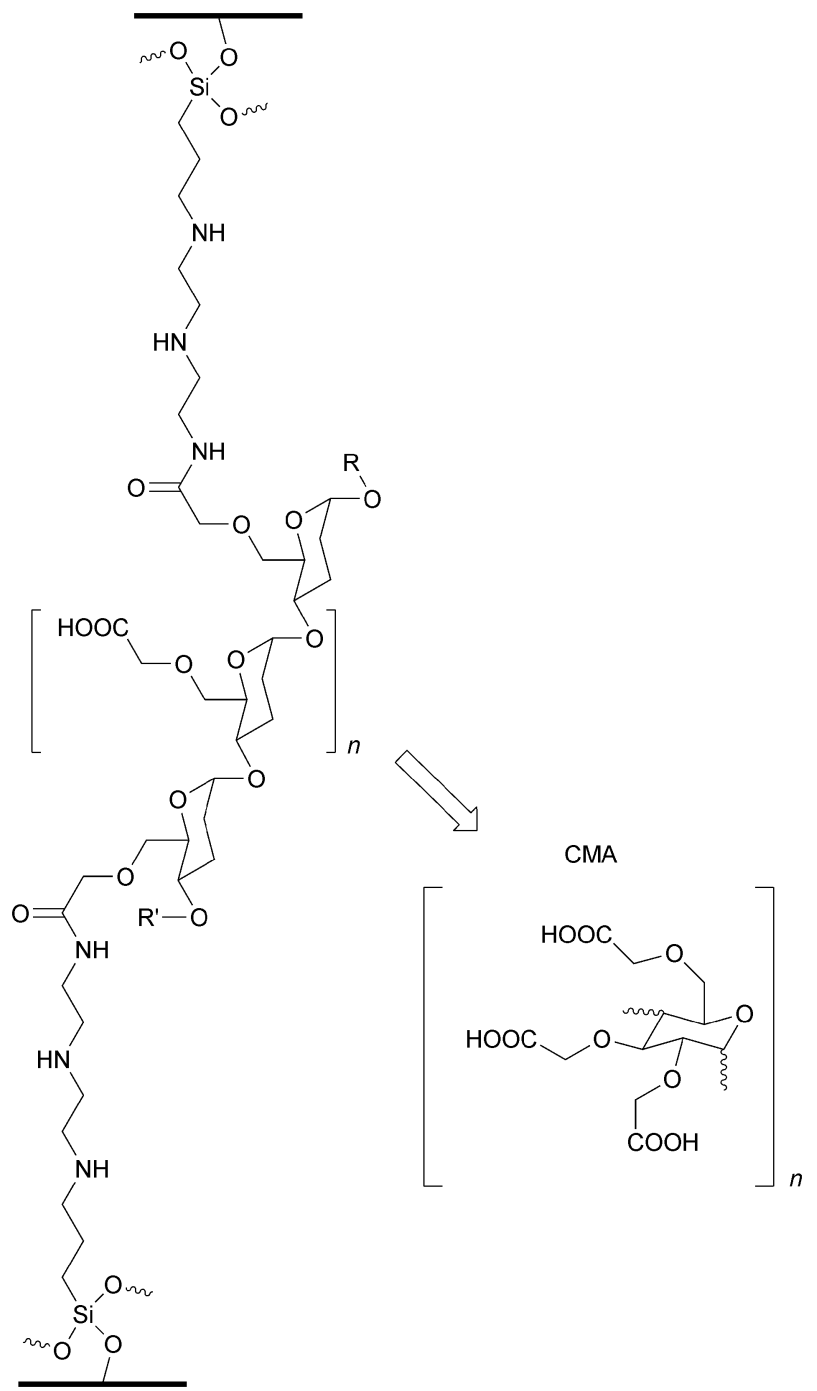

Scheme 1 Single carboxymethylated amylose (CMA) polymer covalently coupled between an amino-functionalized AFM tip and an amino-functionalized glass surface.

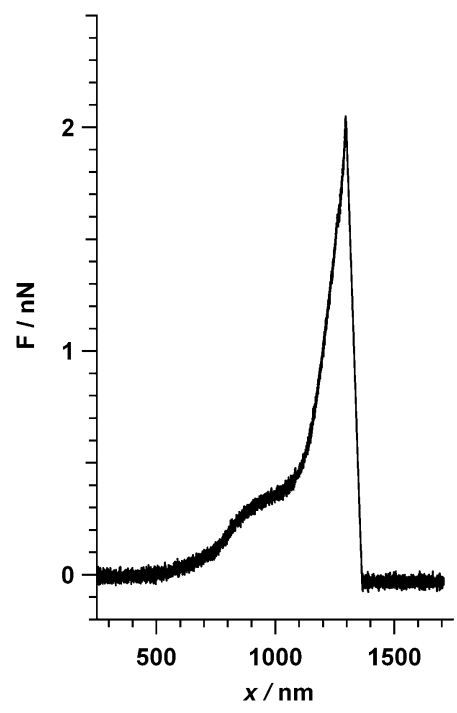

Fig. 1 Typical force-extension curve of a single CMA polymer recorded at $293 \mathrm{~K}$. At low forces, the polymer is uncoiled, then bond angles deform and the chair-boat transition of the glucose rings leads to a pronounced plateau at around $0.3 \mathrm{nN}$. At the end of the plateau, a sharp increase in force can be observed, and at a force of $2.1 \mathrm{nN}$ the connection is finally lost.

plateau at $0.3 \mathrm{nN}$, which is caused by the chair-boat transition of CMA, can be used to confirm that a single molecule has been stretched. At the end of the plateau in Fig. 1, further extension of the molecule leads to a sharp increase in force, until the chain connecting the glass substrate and the AFM cantilever finally breaks at a force of $2.1 \mathrm{nN}$, in this individual example. Based on quantum-chemical modelling, it has been shown that under gas-phase conditions, the weakest link in the chain connecting the two surfaces is the $\mathrm{Si}-\mathrm{C}$ bond in the amino surface linker, ${ }^{14,22}$ and the observed rupture of the connection between the two surfaces has therefore been attributed to the scission of the $\mathrm{Si}-\mathrm{C}$ bond.

In Fig. 2 rupture forces $\left(f_{\mathrm{r}}\right)$ and force-loading rates $(\mathrm{d} f / \mathrm{d} t)$ of almost 900 single molecule rupture events (black dots) at three different temperatures are displayed. Fig. 2a shows rupture forces $v s$. force-loading rates of 182 bond rupture events at 282 K. Fig. 2b shows 408 rupture forces vs. force-loading rates at $293 \mathrm{~K}$, and Fig. 2c shows 306 rupture forces $v s$. forceloading rates at $320 \mathrm{~K}$.

To extract the kinetic parameters of the bond rupture process, an Arrhenius kinetics model, with a Morse potential $V(x)=D_{\mathrm{e}}(1-\exp (-\beta x))^{2}$ representing the binding potential was fitted to the data, using the maximum likelihood method. Here, $D_{\mathrm{e}}$ represents the dissociation energy and $1 / \beta$ the relative width of the potential. With the effective potential $V_{\text {eff }}(x)=V(x)-f x$, the force-dependent activation energy $E_{\mathrm{a}}(f)$ entering the Arrhenius equation becomes: ${ }^{22,34}$

$$
E_{\mathrm{a}}(f)=D_{\mathrm{e}}\left\{\sqrt{1-\frac{f}{f_{\max }}}-\frac{f}{f_{\max }} \operatorname{coth}\left(\sqrt{1-\frac{f}{f_{\max }}}\right)\right\}-\frac{\alpha}{2} h \nu,
$$

where $f_{\max }=V_{\max }^{\prime}=1 / 2 D_{\mathrm{e}} \beta$ and the term $\alpha / 2 h \nu$ accounts for the contribution of the zero-point energy ( $c f$. also ESI $\dagger$ ). 

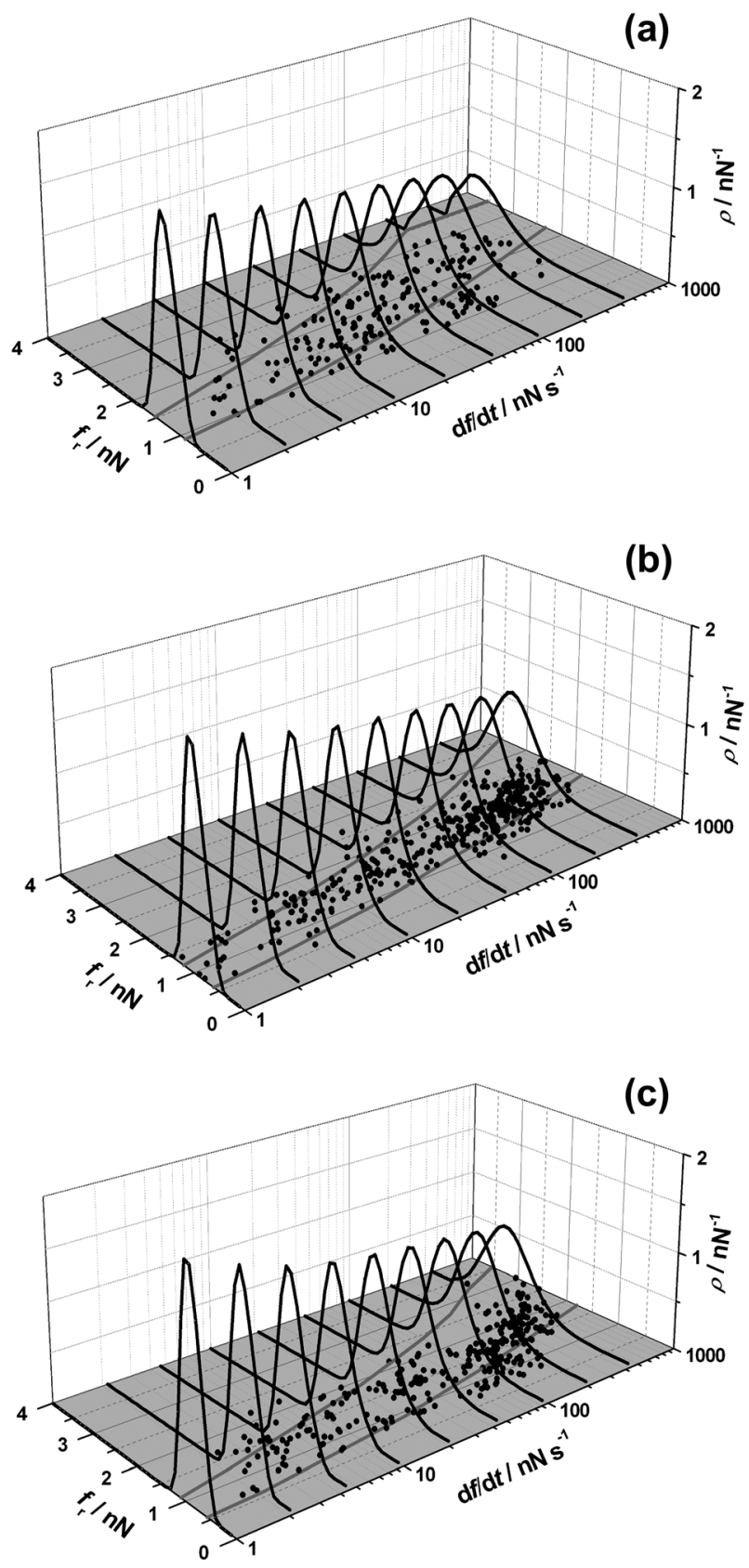

Fig. 2 Bond rupture forces $\left(f_{\mathrm{r}}\right) v s$. force-loading rates $(\mathrm{d} f / \mathrm{d} t)$ of more than 900 single molecule rupture events at $282 \mathrm{~K}$ (a), $293 \mathrm{~K}$ (b), and $320 \mathrm{~K}$ (c). Every data point corresponds to one individual rupture event. The solid lines represent force distributions at force-loading rates between $1 \mathrm{nN} \mathrm{s}^{-1}$ and $464 \mathrm{nN} \mathrm{s}^{-1}$ calculated with the fit parameters $D_{\mathrm{e}}=35.5 \mathrm{~kJ} \mathrm{~mol}^{-1}, f_{\max }=3.55 \mathrm{nN}$, and $A(282 \mathrm{~K})=9.0 \times 10^{2} \mathrm{~s}^{-1}$, $A(293 \mathrm{~K})=3.6 \times 10^{3} \mathrm{~s}^{-1}$, and $A(320 \mathrm{~K})=3.3 \times 10^{3} \mathrm{~s}^{-1}$, respectively. The parameters were obtained by fitting an Arrhenius kinetics model combined with a Morse potential as an analytic representation of the binding potential using the maximum likelihood estimate method.

Based on this activation energy, the force-loading ratedependent rupture force distribution

$$
p_{\mathrm{r}}(f, \dot{f}, T)=-\frac{\mathrm{d}}{\mathrm{d} f} \exp \left\{\frac{A}{\mathrm{~d} f / \mathrm{d} t} \int_{f_{0}}^{f} \exp \left[-E_{\mathrm{a}}(\hat{f}) / k_{\mathrm{B}} T\right] \mathrm{d} \hat{f}\right\}
$$

was numerically calculated for all $\mathrm{N}$ data points at all three temperatures, and the log-likelihood function ${ }^{29}$

$$
\log L\left(A, D_{\mathrm{e},} f_{\max }\right)=\sum_{i=1}^{N} \log p_{\mathrm{r}}\left(f_{i} \dot{f}_{i}, T_{i} \mid A, D_{\mathrm{e},} f_{\max }\right)
$$

was optimized with respect to the three free parameters $A, D_{\mathrm{e}}$, and $f_{\max }$. In the fitting procedure, $D_{\mathrm{e}}$ and $f_{\max }$ were treated as global parameters, because these are structural parameters of the binding potential which should not be affected by temperature, while the Arrhenius pre-factor was determined for each temperature individually, because it may be affected by temperature dependent parameters, such as viscous damping in the buffer solution. (For details of the fitting procedure, $c f$. ESI. $\dagger$ )

In addition to the rupture forces and force-loading rates in the $x-y$-plane, nine exemplary rupture force distributions per temperature have been visualized in Fig. 2 (black curves). As pointed out above, the displayed distributions are based on optimal values for the three fit parameters. The grey lines in the $x-y$ plane represent the boundaries of the $90 \%$ confidence interval of the calculated distributions, i.e. about $10 \%$ of all data points can be expected to lie outside of this range.

The parameters providing the best fit for all three temperatures are $D_{\mathrm{e}}=35.5 \mathrm{~kJ} \mathrm{~mol}^{-1}$, and $f_{\max }=3.55 \mathrm{nN}$. The corresponding Arrhenius pre-factors are $A=9.0 \times 10^{2} \mathrm{~s}^{-1}$ at $282 \mathrm{~K}, A=3.6 \times 10^{3} \mathrm{~s}^{-1}$ at $293 \mathrm{~K}$, and $A=3.3 \times 10^{3} \mathrm{~s}^{-1}$ at $320 \mathrm{~K}$. In Table 1 these values are summarized and compared to values of the $\mathrm{Si}-\mathrm{C}$ bond as determined by gas-phase quantum chemical calculations. With $35.5 \mathrm{~kJ} \mathrm{~mol}^{-1}$, the dissociation energy $D_{\mathrm{e}}$ is almost one order of magnitude smaller than the theoretical gas-phase value of the $\mathrm{Si}-\mathrm{C}$ bond. The $3.55 \mathrm{nN}$ of the maximum force $f_{\max }$ is about $25 \%$ smaller and the Arrhenius $A$-factors are about 10 orders of magnitude smaller than the theoretical gas-phase values for the $\mathrm{Si}-\mathrm{C}$ bond. A smaller value for $A$ might be expected, as the theoretical value represents merely the $\mathrm{Si}-\mathrm{C}$ bonds optical phonon frequency in the gas phase. Viscous damping should reduce this frequency considerably in solution. Furthermore the accommodation coefficient $\kappa$, as well as the ratio of the partition functions of the activated complex and the initial

Table 1 Parameters extracted from experimental data and from DFT-calculations for the weakest bond in the linker, i.e. the $\mathrm{Si}-\mathrm{C}$ bond

\begin{tabular}{llll}
\hline Parameter $^{a}$ & Experimental data $^{b}$ & $\mathrm{Si}-\mathrm{C}$ bond $^{c}$ & Temperature \\
\hline$D_{\mathrm{e}} / \mathrm{kJ} \mathrm{mol}^{-1}$ & 35.5 & 337.4 & Global \\
$f_{\max } / \mathrm{nN}$ & 3.55 & 4.78 & Global \\
$A^{d} / \mathrm{s}^{-1}$ & & $1 \times 10^{13}-5 \times 10^{13}$ & Global \\
& $9.0 \times 10^{2} \mathrm{~s}^{-1}$ & & $282 \mathrm{~K}$ \\
& $3.6 \times 10^{3} \mathrm{~s}^{-1}$ & & $293 \mathrm{~K}$ \\
& $3.3 \times 10^{3} \mathrm{~s}^{-1}$ & & $320 \mathrm{~K}$ \\
$E_{\mathrm{a}}(0)^{e} / \mathrm{kJ} \mathrm{mol}^{-1}$ & 34.0 & & Global
\end{tabular}

${ }^{a}$ Free parameters of the Arrhenius kinetics model combined with a Morse potential. ${ }^{b}$ Values extracted from the global fit to the experimental data shown in Fig. 2a-c. ${ }^{c}$ Results from DFT-calculations in the gasphase. ${ }^{d}$ In the gas-phase, the maximum frequency of an optical phonon in a one-dimensional polymer ${ }^{22}$ was chosen. ${ }^{e}$ The height of the activation barrier at zero force $E_{\mathrm{a}}(O)$ equals the dissociation energy of the Morse potential minus the contribution from the zero-point correction: $E_{\mathrm{a}}(0)=D_{\mathrm{e}}-\alpha / 2 h \nu(c f$. also ESI $\dagger)$. 
state $q^{\ddagger} / q$, both of which are typically smaller than 1 , are not yet included in the gas phase value. ${ }^{18,22,34}$ However, it seems unlikely that this can account for a reduction of 10 orders of magnitude. Together with the severely reduced dissociation energy and the slightly reduced maximum force, these parameters clearly point to a different rupture mechanism than homolytic bond scission.

An alternative path, which agrees much better with the observed kinetic parameters than homolytic bond scission, is the force-induced hydrolysis of a covalent bond. A mechanically activated chemical reaction, like force-induced hydrolysis, could not only explain a significant reduction of the activation energy, but at the same time the fact that reactants, such as protons, hydroxyl ions or water molecules, have to diffuse to reach their reaction partners could explain a reduction of the Arrhenius pre-factor of several orders of magnitude. First principle quantum chemical calculations have demonstrated that mechanical stress can indeed change the proton affinity of various reactive groups and thus trigger chemical reactions, like hydrolysis, which would not occur in the relaxed molecule under the same environmental conditions. ${ }^{23,24}$

As pointed out in previous studies, ${ }^{14,18}$ small microruptures, which are frequently observed in the force-distance curves of the amino-linked CMA, clearly indicate that a bond within the surface linker is indeed breaking in this experimental system. Within this surface linker, the carboxylic acid amide bond $(\mathrm{C}(\mathrm{O})-\mathrm{N})$, and the siloxane bond $(\mathrm{Si}-\mathrm{O})$ are both hydrolysable in aqueous environments. Without mechanical stress, hydrolysis of these bonds requires rather harsh reaction conditions, such as extreme $\mathrm{pH}$ values and elevated temperatures. Experimentally determined activation energies for the amide bond lie in the range between $76 \mathrm{~kJ} \mathrm{~mol}^{-1}$ and $105 \mathrm{~kJ} \mathrm{~mol}^{-1},{ }^{35-39}$ the corresponding values for the siloxane bond are between $67 \mathrm{~kJ} \mathrm{~mol}^{-1}$ and $96 \mathrm{~kJ} \mathrm{~mol}^{-1} \cdot 35,40-42$ Theoretical values are $63-113 \mathrm{~kJ} \mathrm{~mol}^{-1}$ for the amide ${ }^{43-49}$ and $71-142 \mathrm{~kJ} \mathrm{~mol}^{-1}$ for the siloxane bond. ${ }^{50-54}$ Bershtein et al., who have studied the mechanically activated hydrolysis of $\mathrm{C}(\mathrm{O})-\mathrm{N}$ and $\mathrm{Si}-\mathrm{O}$ bonds in bulk material in moisture, have found activation energies between $84 \mathrm{~kJ} \mathrm{~mol}^{-1}$ and $105 \mathrm{~kJ} \mathrm{~mol}^{-1}$ for $\mathrm{C}(\mathrm{O})-\mathrm{N}$ and $80 \mathrm{~kJ} \mathrm{~mol}^{-1}-96 \mathrm{~kJ} \mathrm{~mol}^{-1}$ for $\mathrm{Si}-\mathrm{O} .{ }^{35}$ However, as pointed out above, all these studies have been carried out under very different experimental conditions (extreme $\mathrm{pH}$ and temperature, etc.). Only Bershtein et al. have actually considered the role of mechanical stress. They found that bond hydrolysis is strongly activated by tensile stress. Nevertheless, their experiments were carried out using bulk material in a gas atmosphere with only $3-4 \%$ humidity in the case of $\mathrm{C}(\mathrm{O})-\mathrm{N}$ and less than $1 \%$ in the case of $\mathrm{Si}-\mathrm{O}$. For these reasons, it is rather difficult to directly compare our results, which have been obtained in aqueous environment at neutral $\mathrm{pH}$ to these data and to determine which bond is actually breaking in our experiments. It should be noted however, that according to the available experimental data, not only the activation energy for hydrolysis of the siloxane bond is slightly lower than the activation energy of the amide bond, but with a bond length of $1.77 \AA$, the $\mathrm{Si}-\mathrm{O}$ bond is also longer than the $\mathrm{C}-\mathrm{N}$ bond with only $1.47 \AA$, ${ }^{55}$ which would make the $\mathrm{Si}-\mathrm{O}$ bond more susceptible to mechanical stress.
Although the parameters in Table 1 correspond to the best fit of an Arrhenius kinetics model with a one-dimensional Morse potential representing the binding potential, the rupture force distributions and the confidence intervals shown in Fig. 2 do not perfectly coincide with the experimental data: at low force-loading rates, the observed rupture forces tend to be slightly higher than the fit, while at high force-loading rates, they tend to be lower than the fit. In fact at $282 \mathrm{~K}$ only $63 \%$ of the data points are within the $90 \%$ confidence interval. At $293 \mathrm{~K} 85 \%$ and at $320 \mathrm{~K} 75 \%$ of the experimental data points are within the $90 \%$ confidence interval of the calculated rupture force distributions. Nevertheless, unlike a least square fit to the most probable or the mean rupture force, our fit algorithm uses not only one point, e.g. the maximum of the rupture force distribution, but it takes into account the exact shape of the distribution for each temperature and force-loading rate. As a consequence, the slope of the rupture forces in the force $v s$. force-loading rate plane is correlated to the width of the distributions: a large slope corresponds to a narrow binding potential, which in turn corresponds to a wide rupture force distribution, i.e. a large scattering of rupture forces. ${ }^{56}$ Apparently, slope and scattering of the experimental data are not perfectly consistent with a simple onedimensional Morse potential based Arrhenius kinetics model, and the fit parameters represent a compromise between the optimal slope in the force $v s$. force-loading rate plane and optimal width of the fitted distributions.

To check whether experimental errors could explain the discrepancy between experimental data and theoretical model, we have included an additional statistical error of $\pm 0.1 \mathrm{nN}$ into the model (data not shown). However, even with an experimental error of $\pm 0.1 \mathrm{nN}$, the quality of the fit can be hardly improved: the moderate slope at low force-loading rates and the much higher slope at high force-loading rates can still not be accurately accounted for by the model.

We therefore believe that a one-dimensional Morse potential with a single decaying bond is just not adequately representing our experimental system. According to a recent paper by Iozzi et al. ${ }^{28}$ where several analytical binding potentials have been compared to $a b$ initio quantum chemical modelling, a Morse potential represents chemical binding potentials reasonably well. Even though, it is not clear whether this is also the case for the hydrolysis of chemical bonds. On the other hand, in our experimental setup, the carboxylic acid amide and the siloxane bond are loaded in series and, as pointed out above, their activation energies are comparable. Therefore another explanation for the systematic discrepancy between the kinetic model and the experimental data could be the presence of two alternative paths by which chain scission can occur, i.e. two different hydrolysable bonds in the surface anchor. If the two bonds were comparable in strength, it would be statistically determined which one of the two bonds in series is actually breaking in any particular rupture experiment.

However, if the amide bond is somewhat stronger under mechanical load, as proposed by the data of Bershtein et al., ${ }^{35}$ it is still possible that our data consist of two different subsets, belonging to two different rupture mechanisms: the aminosilane surface linker can be connected to the glass substrate via one, two, or three silanol groups. Several parallel connections 
would obviously strengthen its connection to the substrate considerably. ${ }^{52}$ In this case, the $\mathrm{C}(\mathrm{O})-\mathrm{N}$ bond might rupture first, although according to Bershtein et al., it has the higher activation energy. Nevertheless, if there is only one silanol link to the substrate, which is most likely the case quite frequently, ${ }^{57,58}$ the $\mathrm{Si}-\mathrm{O}$ bond might rupture first, due to its lower activation energy. ${ }^{35}$ In any event, different surface anchoring will lead to different rupture kinetics, which will lead to a broadening of the rupture force distributions. First force-clamp experiments carried out in our lab indicate that this may in fact be the case.

It should be pointed out that the exact values of the kinetic parameters depend, of course, on the kinetic model. The Arrhenius kinetics model used here seems to be too simplistic to perfectly explain all details of the experimental data. However, the large changes in rupture forces, upon relatively small changes on the (absolute) temperature scale, can only be accounted for by low activation energy. This agrees much better with mechanically activated hydrolysis than with homolytic bond scission.

\section{Conclusions}

In the present study, we have identified structural and kinetic parameters for the mechanically activated scission of single covalent bonds in aqueous environment with dynamic single molecule force spectroscopy, using temperature as an additional control parameter. We found a significantly smaller dissociation energy and Arrhenius pre-factor, than expected for homolytic bond scission. One possible explanation for the severely reduced dissociation energy is the mechanically activated hydrolysis of chemical bonds, like the carboxylic acid amide or the siloxane bond in the amino-silane surface linker. To clarify whether hydrolysis is indeed the correct rupture mechanism and if so, which bonds are actually hydrolysed, further quantum chemical studies of these bonds under mechanical stress, which also incorporate water as a solvent, will have to be carried out using new methods like an explicitly included external force field (EFEI). ${ }^{27,59,60}$ Force-clamp experiments have to show whether the reactive ensemble is composed of one or more subsets which differ in the details of the surface anchoring. Such an effect would also lead to a broadened rupture force distribution.

\section{Experimental}

\section{Materials}

$N^{1}$-[3-(Trimethoxysilyl)-propyl]diethylenetriamine (DETA), carboxymethylated amylose (CMA), polymer bound 1-(3-dimethylaminopropyl)-3-ethylcarbodiimide (EDC), $N$-hydroxysuccinimide (NHS), and phosphate buffered saline (PBS; buffer composed of $0.137 \mathrm{M} \mathrm{NaCl}, 0.010 \mathrm{M} \mathrm{Na}_{2} \mathrm{HPO}_{4}, 0.003 \mathrm{M} \mathrm{KCl}$, and $0.002 \mathrm{M} \mathrm{KH}_{2} \mathrm{PO}_{4}, \mathrm{pH} 7.4$ at $T=25^{\circ} \mathrm{C}$ ) were purchased from Sigma-Aldrich (Deisenhofen, Germany). Hydrochloric acid (32\% GR for analysis), acetic acid (99-100\% for synthesis), and ethanol (absolute GR for analysis) were obtained from Merck (Darmstadt, Germany). All experiments were conducted with silicon nitride AFM cantilevers with a nominal force constant between 10 and $20 \mathrm{mN} \mathrm{m}^{-1}$ (MLCT-AU, Veeco Instruments $\mathrm{GmbH}$, Mannheim, Germany, and OMCL-TR400PSA, Olympus Corp., Tokyo, Japan). Glass microscope slides from Menzel (Braunschweig, Germany) were used as substrates.

\section{Sample preparation}

Glass microscope slides were cleaned in a $2.5 \% \mathrm{HCl}$ solution in double distilled water (henceforth referred to as $\mathrm{H}_{2} \mathrm{O}_{\text {ddest }}$ ) for $120 \mathrm{~min}$, sonicated in this solution for $60 \mathrm{~min}$, and rinsed three times in $\mathrm{H}_{2} \mathrm{O}_{\text {ddest }}$. To remove organic contaminations from the AFM tip, cantilevers were irradiated with UV light over $60 \mathrm{~min}$ and immersed in ethanol. To amino-functionalize the surfaces, a $10: 1$ solution of ethanol and $\mathrm{H}_{2} \mathrm{O}_{\text {ddest }}$ was prepared and adjusted to $\mathrm{pH} 4.5-5.5$ with diluted acetic acid. Then, DETA was added to the acetic ethanol $/ \mathrm{H}_{2} \mathrm{O}_{\text {ddest }}$ solution to a final concentration of $2 \%$, and glass slides and AFM cantilevers were incubated for $60 \mathrm{~min}$ in this solution, rinsed with ethanol, and cured at $110{ }^{\circ} \mathrm{C}$ for $20 \mathrm{~min}$.

Prior to individual force-spectroscopy experiments $10 \mathrm{mg} \mathrm{mL}^{-1}$ CMA, $10 \mathrm{mg} \mathrm{mL}^{-1} \mathrm{EDC}$, and $1 \mathrm{mg} \mathrm{mL}^{-1}$ NHS were solved in $1 \mathrm{~mL}$ PBS. The solution was then transferred to the aminosilanized microscope slide. After a reaction period of $10 \mathrm{~min}$ the slide was rinsed thoroughly with PBS in order to remove non-covalently bound CMA from the slide.

\section{Experimental setup}

Prepared slides were immediately mounted on a custom-built AFM stage and covered with several hundred $\mu \mathrm{L}$ of PBS. The temperature of the solution was controlled by a thermostat with a temperature constancy of $\pm 0.03{ }^{\circ} \mathrm{C}$ (CF30 KryoKompakt-Thermostat, Julabo Labortechnik GmbH, Seelbach, Germany). The thermostat was connected with a fluid cooler (FLKU 140 G 200, Fischer Elektronik GmbH, Lüdenscheid, Germany), which was mounted on the AFM stage serving as slide holder. Prior to AFM measurements the temperature of the solution on the stage was adjusted and after equilibrium was achieved, it was kept constant during experiments. Afterwards, the amino-functionalized AFM tip was brought into contact with the activated CMA on the glass slide, and force-piezo-distance curves were recorded with the AFM (NanoWizard, JPK Instruments, Berlin, Germany) in the force spectroscopy mode. In order to obtain a higher binding rate the contact time before retraction of the tip was set to $0.5 \mathrm{~s}$. To cover a wide range of force-loading rates the $z$-piezo velocities were varied between $100 \mathrm{~nm} \mathrm{~s}^{-1}$ and $50000 \mathrm{~nm} \mathrm{~s}^{-1}$.

After the recording of the force curves, the optical lever sensitivity and cantilever force constant were determined threefold for each cantilever individually using the thermal noise method. ${ }^{61}$ The average value of the three runs was then used to calibrate the force-distance curves and extract bond-rupture forces and force-loading rates. To minimize scattering due to calibration errors all force-distance curves were normalized with respect to the force at the inflection point on the plateau for every temperature individually.

The bond-rupture forces correspond to the maximum force before the bond ruptures and the cantilever snaps back to zero force. The effective spring constant of the cantilever and 
polymer was determined from the slope of the forcepiezo-distance curve before the bond ruptures, and multiplied with the piezo-velocity in order to extract the force-loading rate. To convert force-piezo-distance curves into force-extension-curves the cantilever deflection was subtracted ( $c f$. also ref. 18).

\section{Acknowledgements}

S.W.S. and H.C.-S. gratefully acknowledge financial support of the German Excellence Initiative via the "Nanosystems Initiative Munich (NIM)". M.K.B. gratefully acknowledges startup funds from the Sonderforschungsbereich 677 "Function by Switching" supported by the Deutsche Forschungsgemeinschaft.

\section{References}

1 O. Schwarz and F.-W. Ebeling, Kunststoffkunde, Vogel Fachbuchverlag, Würzburg, 2004.

2 A. M. Dubinskaya, Usp. Khim., 1999, 68, 708-724.

3 V. V. Boldyrev and K. Tkacova, J. Mater. Synth. Process., 2000, 8 , 121-132.

4 M. K. Beyer and H. Clausen-Schaumann, Chem. Rev., 2005, 105, 2921-2948.

5 C. R. Hickenboth, J. S. Moore, S. R. White, N. R. Sottos, J. Baudry and S. R. Wilson, Nature, 2007, 446, 423-427.

6 D. A. Davis, A. Hamilton, J. L. Yang, L. D. Cremar, D. Van Gough, S. L. Potisek, M. T. Ong, P. V. Braun, T. J. Martinez, S. R. White, J. S. Moore and N. R. Sottos, Nature, 2009, 459, 68-72.

7 J. Liang and J. M. Fernandez, ACS Nano, 2009, 3, 1628-1645.

8 M. M. Caruso, D. A. Davis, Q. Shen, S. A. Odom, N. R. Sottos, S. R. White and J. S. Moore, Chem. Rev., 2009, 109, 5755-5798.

9 E. L. Florin, V. T. Moy and H. E. Gaub, Science, 1994, 264, 415-417.

10 V. T. Moy, E. L. Florin and H. E. Gaub, Science, 1994, 266, 257-259.

11 P. Hinterdorfer, W. Baumgartner, H. J. Gruber, K. Schilcher and H. Schindler, Proc. Natl. Acad. Sci. U. S. A., 1996, 93, 3477-3481.

12 H. Clausen-Schaumann, M. Seitz, R. Krautbauer and H. E. Gaub, Curr. Opin. Chem. Biol., 2000, 4, 524-530.

13 B. M. Gaub, C. Kaul, J. L. Zimmermann, T. Carell and H. E. Gaub, Nanotechnology, 2009, 20, 434002-434009.

14 M. Grandbois, M. Beyer, M. Rief, H. Clausen-Schaumann and H. E. Gaub, Science, 1999, 283, 1727-1730.

15 M. A. Lantz, H. J. Hug, R. Hoffmann, P. J. A. van Schendel, P. Kappenberger, S. Martin, A. Baratoff and H. J. Guntherodt, Science, 2001, 291, 2580-2583.

16 P. Schwaderer, E. Funk, F. Achenbach, J. Weis, C. Bräuchle and J. Michaelis, Langmuir, 2007, 24, 1343-1349.

17 A. P. Wiita, S. R. K. Ainavarapu, H. H. Huang and J. M. Fernandez, Proc. Natl. Acad. Sci. U. S. A., 2006, 103, $7222-7227$.

18 S. W. Schmidt, M. K. Beyer and H. Clausen-Schaumann, J. Am. Chem. Soc., 2008, 130, 3664-3668.

19 N. V. Lebedeva, F. C. Sun, H. I. Lee, K. Matyjaszewski and S. S. Sheiko, J. Am. Chem. Soc., 2008, 130, 4228-4229.

20 S. Garcia-Manyes, J. Liang, R. Szoszkiewicz, T.-L. Kuo and J. M. Fernandez, Nat. Chem., 2009, 1, 236-242.

21 I. Park, D. Shirvanyants, A. Nese, K. Matyjaszewski, M. Rubinstein and S. S. Sheiko, J. Am. Chem. Soc., 2010, 132, 12487-12491.

22 M. K. Beyer, J. Chem. Phys., 2000, 112, 7307-7312.

23 D. Aktah and I. Frank, J. Am. Chem. Soc., 2002, 124, 3402-3406.

24 M. K. Beyer, Angew. Chem., Int. Ed., 2003, 42, 4913-4915.
25 M. Konopka, R. Turansky, J. Reichert, H. Fuchs, D. Marx and I. Stich, Phys. Rev. Lett., 2008, 100, 1155031-1155034.

26 U. Lourderaj, J. L. McAfee and W. L. Hase, J. Chem. Phys., 2008, 129, 0947011-09470110.

27 J. Ribas-Arino, M. Shiga and D. Marx, Angew. Chem., Int. Ed., 2009, 48, 4190-4193.

28 M. F. Iozzi, T. Helgaker and E. Uggerud, Mol. Phys., 2009, 107, 2537-2546.

29 S. Brandt, Data Analysis: Statistical and Computational Methods for Scientists and Engineers, Springer Verlag, Berlin, 1998

30 M. Rief, F. Oesterhelt, B. Heymann and H. E. Gaub, Science, 1997, 275, 1295-1297.

31 P. E. Marszalek, A. F. Oberhauser, Y. P. Pang and J. M. Fernandez, Nature, 1998, 396, 661-664.

32 H. B. Li, M. Rief, F. Oesterhelt, H. E. Gaub, X. Zhang and J. C. Shen, Chem. Phys. Lett., 1999, 305, 197-201.

33 B. Heymann and H. Grubmüller, Chem. Phys. Lett., 1999, 305, 202-208.

34 F. Hanke and H. J. Kreuzer, Phys. Rev. E: Stat., Nonlinear, Soft Matter Phys., 2006, 74, 0319091-0319095.

35 V. A. Bershtein, L. M. Egorova and V. V. Solov'ev, Mech. Compos. Mater., 1977, 13, 715-721.

36 B. A. Robinson and J. W. Tester, Int. J. Chem. Kinet., 1990, 22, 431-448.

37 H. Slebocka-Tilk, F. Sauriol, M. Monette and R. S. Brown, Can. J. Chem., 2002, 80, 1343-1350.

38 A. Meyer, N. Jones, Y. Lin and D. Kranbuehl, Macromolecules, 2002, 35, 2784-2798.

39 T. M. Alam, Polymer, 2003, 44, 6531-6536.

40 W. Hertl, J. Phys. Chem., 1968, 72, 3993-3997.

41 J. D. Rimstidt and H. L. Barnes, Geochim. Cosmochim. Acta, 1980, 44, 1683-1699.

42 P. M. Dove and D. A. Crerar, Geochim. Cosmochim. Acta, 1990, 54, 955-969.

43 J. P. Guthrie, J. Am. Chem. Soc., 1974, 96, 3608-3615.

44 D. Bakowies and P. A. Kollman, J. Am. Chem. Soc., 1999, 121, $5712-5726$.

45 M. Cascella, S. Raugei and P. Carloni, J. Phys. Chem. B, 2004, 108, 369-375.

46 D. Zahn, Chem. Phys. Lett., 2004, 383, 134-137.

47 J. R. Pliego, Chem. Phys., 2004, 306, 273-280.

48 J. Blumberger and M. L. Klein, Chem. Phys. Lett., 2006, 422, 210-217.

49 Y. Xiong and C. G. Zhan, J. Phys. Chem. A, 2006, 110, $12644-12652$.

50 Y. T. Xiao and A. C. Lasaga, Geochim. Cosmochim. Acta, 1994, 58, $5379-5400$.

51 Y. T. Xiao and A. C. Lasaga, Geochim. Cosmochim. Acta, 1996, 60, 2283-2295.

52 A. Pelmenschikov, H. Strandh, L. G. M. Pettersson and J. Leszczynski, J. Phys. Chem. B, 2000, 104, 5779-5783.

53 M. Cypryk and Y. Apeloig, Organometallics, 2002, 21, 2165-2175.

54 J. E. Del Bene, K. Runge and R. J. Bartlett, Comput. Mater. Sci., 2003, 27, 102-108.

55 A. F. Hollemann and E. Wiberg, Hollemann-Wiberg Lehrbuch der anorganischen Chemie, Walter de Gruyter, Berlin, New York, 1995.

56 O. K. Dudko, G. Hummer and A. Szabo, Phys. Rev. Lett., 2006, 96, 1081011-1081014.

$57 \mathrm{~S}$. Iarlori, D. Ceresoli, M. Bernasconi, D. Donadio and M. Parrinello, J. Phys. Chem. B, 2001, 105, 8007-8013.

58 S. W. Schmidt, T. Christ, C. Glockner, M. K. Beyer and H. Clausen-Schaumann, Langmuir, 2010, 26, 15333-15338.

59 J. Ribas-Arino, M. Shiga and D. Marx, Chem.-Eur. J., 2009, 15, 13331-13335.

60 J. Ribas-Arino, M. Shiga and D. Marx, J. Am. Chem. Soc., 2010, 132, 10609-10614.

61 H.-J. Butt and M. Jaschke, Nanotechnology, 1995, 6, 1-7. 